\title{
What is learned: All kinds of things
}

\author{
KENNETH R. BURSTEIN \\ Simon Fraser University, Burnaby, British Columbia V5A 1S6, Canada
}

\begin{abstract}
An attempt is made to demonstrate that it is fruitless to attempt to define or restrict the types of relationships that can be learned on the basis of categories (e.g., "stimuli," "responses") that have no relationship whatsoever to the functional characteristics of the neural system mediating the experience of such relationships.
\end{abstract}

Bolles (1972) asserts that "what is learned is a direct appreciation of the new environmental contingencies" (p. 402). This reiteration that learning involves the perception of relationships is not unwelcome, although it might be fruitful to acknowledge that learning also seems to involve, to use Bolles' term, an "appreciation" of the reliability of these relationships. Perhaps his statement should be revised to read: "What is learned is a direct appreciation of the degree of correlation between events."

Bolles (1972) goes on, however, to suggest that there are two kinds of contingencies, or expectancies, S-S*1 and R-S*, and that these "constitute all of what is learned in most instrumental learning experiments" (p. 403). This assertion of two exhaustive laws of learning, reflecting two exhaustive kinds of learning, the first involving a contingency between a stimulus and a reinforcement and the second involving a contingency between a response and a reinforcement, returns to the psychological literature a red herring that has been floundering around for decades. What follows here is an attempt to demonstrate that the elements, or events, between which contingencies or relationships are hypothesized cannot be restricted to particular arbitrarily selected subsets of a language system when the terms of the language system are equivalent and indistinguishable to the neurophysiological substrate that mediates the perception of these contingencies or relationships.

This is not to say that psychological theories must be expressed in or translatable into neurophysiological terms. However, psychological theories, like all theories, should not contradict known facts, whatever the language or level of the facts. What follows, then, is not an argument for "reductionism," but a plea that time not be wasted on positions that are contradicted by available information.

First, the appreciation of contingencies or relationships is acknowledged by most to be mediated via the nervous system. Bolles (1972) notes that he does "not wish to conjecture either about its localization in the nervous system or to what extent it may be represented

This paper was supported by Grant APA-193 from the National Research Council of Canada. in consciousness" (p. 403). Although this would appear to be a wise strategy, there may be some value to addressing not the question of localization, but rather the question of the characteristics of the mediating nervous system.

That is, if relationships are what is learned, which seems quite reasonable, those relationships are perceived or appreciated either at the sensory level (which may be what Bolles, 1972, means by "directly appreciated") or by some postsensory but premotor portion of the nervous system.

In the former case, it should be obvious that the labels "stimuli," "responses," "Ss," "S*s," and "Rs" have no meaning whatsoever with respect to the functioning of the sensory systems. The appreciation of "responses" and their relationship to other events is accomplished by the sensory system in a manner that is indistinguishable, in principle, from the appreciation of "stimuli" and their relationship to other events.

The sensory system makes no distinction between responses and stimuli. It is almost banal to point out that there is no "motor world" and that the term possesses whatever meaning it has only because of the sensory feedback from events that we arbitrarily label as "responses." The afferent systems have no receptors or transducers for distinguishing stimuli from responses. Certain patterns of complex sensory input may be labeled "responses" by a postsensory system, but the sensory system itself does not respond differentially to "stimuli" and "responses."

It should be clear, therefore, that if one of the characteristics of the sensory system is to provide an appreciation not only of events, but also of the relationship between such events, such relationships cannot be restricted on the basis of an arbitrary set of labels that are unrelated to the structure and function of the system mediating them. If the appreciation of relationships is a capacity of the mediating system, such relationships cannot be restricted to "S-S*," "S-R," "S-S," or "R-S*" relationships. The sensory system transmits information about all energy changes that effectively energize it, regardless of whether higher structures label these energy changes "stimuli," "responses," "tables," or "Pontiacs."

Furthermore, even if the sensory systems did possess 
some peripheral mechanism for differentiating energy changes on the basis of an S-R language system, or on any other basis, it is inconceivable that the capacity to perceive or appreciate relationships between these events could be limited to certain sequences of events. That is, if the sensory system could distinguish stimuli from responses, Cadillacs from Pontiacs, how could the capacity for the perception of contingencies apply to stimulus-response relationships and not to responsestimulus relationships? How could a relationship be appreciated between the sound of a starter's gun and running and not be appreciated between running followed by a starter's gun?

In addition, although the number of events hypothesized in Bolles' (1972) paper and in most papers taking similar positions seems typically to be two or three, it seems fairly obvious that the total number of permutated events and the total number of permutations involved in a relationship can be limited only by the capacity of the mediating system, and that capacity is much more than two or three events. Thus, if the S-R language system is to be employed, relationships must be possible with sequences such as R-R-S, R-R-R, S*-R-R-R, S*-R-R-S*-S, and so on.

One could argue, however, that while it is true that the sensory system cannot distinguish between events on the basis of these labels or restrict the perception of relationships to certain sequences of events, there is some postsensory system that is able to do these things.

Organisms certainly can and do, through experience and learning, label certain sensory experiences "stimuli" and others "responses." Nevertheless, if the appreciation of relationships between events is a capacity of the sensory system, the ability of a postsensory system to apply labels to the events within these relationships does not alter the above argument.

One could also argue that the capacity to appreciate relationships resides not in the sensory system, but in the same system that is capable of labeling sensory events. This argument also does not affect the conclusion presented above, since the capacity to appreciate relationships, a capacity inherent in the system, cannot be altered by any arbitrary labeling of the events being appreciated. While it is clearly probable that attentional and motivational variables may operate so as to reduce or to enhance the appreciation or retention of certain types of relationships, it seems obvious that such effects are not limited to relationships other than those of the S-S* or R-S* variety, but they occur to varying degrees in all possible classes of relationships that can be defined by this language system.

While this comment may appear unfair or unjustified because some positions specifically note that the relationships hypothesized apply only to certain learning procedures (e.g., Bolles', 1972, assertion that the $\mathrm{S}^{*}$ is the terminal event learned in instrumental procedures), the acceptance of this type of restriction reflects a confusion with respect to the learning-performance distinction.

Thus, it should be apparent that the $\mathrm{S}^{*}$ mentioned by Bolles (1972) is the terminal event in instrumental conditioning procedures, not because contingencies of forms other than R-S* and S-S* are not learned or "appreciated," but because the instrumental procedure is structured so that $\mathrm{S}^{*}$ is the only terminal event possible. There is no provision within the procedure for any performance reflecting the learning or "appreciation" of forms other than S-S* and R-S*.

Contingencies or correlations are learned because some of them are useful. A correlation between a tone (or twig breaking) and a dinosaur (CS-UCS) is useful, since it allows an organism to respond to the dinosaur prior to actual physical encounter. A correlation between dinosaur and tone (UCS-CS) can be utilized in no adaptive way. To say that forward conditioning procedures result in learning and that backward conditioning procedures do not is an error, for organisms that learned or appreciated both of these relationships could only translate one of them into performance. Backward conditioning procedures result in low levels of performance for precisely the same reason that non-S* terminated behaviors do not occur in instrumental procedures: They are not adaptive.

There are obviously many types of contingencies and degrees of contingencies that can be learned, and are learned, within instrumental conditioning experiments and in other situations, but that cannot be demonstrated in a situation designed to allow only certain classes or types of contingencies to manifest themselves. The instrumental conditioning paradigm is simply one technique for obtaining empirical data from which inferences can be made about certain arbitrarily labeled classes of contingencies. In order to assert that these contingencies are the only, or virtually the only, contingencies learned with this particular technique, it is necessary to provide an opportunity for other contingencies to manifest themselves. Until this is done, there is no empirical basis for assuming that other contingencies are not learned.

It may be largely a result of this general acceptance of the view that only certain types of relationships are learned in certain situations that research in highly related areas of learning feed, not into one general theory of learning, but into more or less independent "restricted" theories. While there are occasional attempts to "hammer" findings in, say, rote serial learning into an S-R framework, it should be obvious that the lack of success in such attempts may stem from the fixation on there being only S-R types of learning. This is not an attack on so-called S-R positions, but merely a plea that we not restrict our research to situations that allow only certain kinds of learned relationships to manifest themselves. It seems at least possible that, if we developed techniques for observing the 
learning of all possible kinds of relationships between sensory events, perhaps even abandoning the arbitrary labels that we now attach to these sensory events, a uniformity might emerge that might allow research in classical conditioning, instrumental conditioning, concept formation, problem solving, rote memory, and so on, to feed into the same theory, rather than into the different theories that appear to exist today. Moreover, we virtually outlaw certain kinds of everyday behavior from research, apparently because it does not fit into our fixed preconceptions of what kind of relationships are learned.

Do we not buy, plant, fertilize, water, prune, and spray (all "responses") a rosebush as the result of learning the relationship between these events and a "terminal" event that is clearly the sensory experience of seeing and smelling some roses? Is this not in the dichotomous world of Ss and Rs an R-R-R-R- . . R-S relationship?

The tendency seems to be either to ignore examples such as these or to hammer or mold them into being congruent with some ill-fitting preconception of exhaustive contingency classes.
To modify slightly a hackneyed criticism, we seem to spend our time looking at what goes on under one lamp post when there are many others around, because we have developed a fondness for one lamp post, or because we are comfortable around it, or because we do not care that most behavior occurs under other illumination, or perhaps because the combined light from other sources has blinded us.

\section{REFERENCE}

Bolles, R. C. Reinforcement, expectancy, and learning. Psychological Review, 1972, 79, 394-409.

\section{NOTE}

1. The attempt to define $S^{*}$ s or reinforcers in terms of biological importance seems inadequate, since there are energy changes that are biologically important but are not capable of serving as reinforcers (e.g., the substitution of non-life-sustaining gases for oxygen in the air). It seems more probable that reinforcers are those stimuli that elicit intense affective responses, either positive or negative.

(Received for publication July 8, 1980.) 